\title{
Disruption of latent inhibition by placement of an electrode in the dorsal hippocampus
}

\author{
TERRY L. DeVIETTI, RITA Y. EMMERSON, and TIMOTHY K. WITTMAN \\ Central Washington University, Ellensburg, Washington
}

\begin{abstract}
Rats were implanted unilaterally with electrodes placed in either the dorsal hippocampus or the mesencephalic reticular formation. Subgroups were given either zero or six preexposure trials to a tone that was subsequently paired with a footshock. An additional group of dorsal hippocampal animals was given 12 preexposure trials. Test performance indicated that all groups given preexposure trials, with the exception of the dorsal hippocampal group given six such trials, showed latent inhibition. A second experiment suggested that the disruption of latent inhibition in the dorsal hippocampal animals given six preexposure trials was not due to seizure activity. The implications of these results are discussed.
\end{abstract}

Organisms preexposed to a to-be-conditioned stimulus show a deficit in conditioning performance when that stimulus is subsequently paired with an unconditioned stimulus. This phenomenon has been termed latent inhibition by Lubow and Moore (1959). One variable critical to the demonstration of latent inhibition is the number of preexposure trials administered. Lubow (1973), in a review of the literature, concluded that most studies using fewer than 16-20 preexposure trials failed to obtain latent inhibition, whereas studies using more than 20 preexposure trials were generally successful in demonstrating the effect. Indeed, it is not uncommon to use several hundred preexposure trials in some preparations (e.g., Solomon \& Moore, 1975; Salafia, Note 1).

In preliminary work aimed at establishing parameters to produce latent inhibition in rats with unilateral electrode placement in the mesencephalic reticular formation (MRF), we found that as few as six preexposure trials were sufficient to produce the effect. Subsequently, we tested the idea that this rapid development of latent inhibition might have resulted from the locus of the electrode implant and/or the surgical procedure (DeVietti, Wittman, \& Comfort, 1980). The results of that study indicated that latent inhibition was obtained with six preexposure trials in animals implanted in the MRF, the corpus collosum, or the cerebral cortex, as well as in sham-operated and unoperated controls. However, in three animals, the electrode tip was located in the dorsal hippocampus (DH), and no evidence of latent inhibition was found. Since the sample size was too small for any

This research was supported in part by the small grants program of Central Washington University. We gratefully acknowledge the assistance of Jim Thomson, Mike Hymes, and David Thatcher. Requests for reprints should be sent to Terry L. DeVietti, Psychology Department, Central Washington University, Ellensburg, Washington 98926. meaningful conclusions to be drawn, these data were not reported. In light of several studies showing that extensive lesions in the $\mathrm{DH}$ disrupted latent inhibition (Ackil, Mellgren, Halgren, \& Frommer, 1969; Solomon \& Moore, 1975), we suspected that, under our conditions, the mere presence of an electrode in the DH may have been sufficient to block latent inhibition. We tested this idea in Experiment 1 by comparing the performance of rats unilaterally implanted in either the MRF or the DH in a latent inhibition paradigm in which either zero or six preexposure trials were administered.

\section{EXPERIMENT 1}

\section{Method}

Animals and Apparatus. The animals were 52 male Long-Evans rats from the Central Washington University psychology colony, aged 90-120 days at the beginning of the experiment. The basic apparatus has been described in detail elsewhere (DeVietti \& Larson, 1971). Two identical chambers were used for the preexposure, fear conditioning, and testing phases of the experiment. They measured $20 \times 30 \mathrm{~cm}$ and were $30 \mathrm{~cm}$ high. The sides and top were Plexiglas, while the front and back were aluminum painted flat gray. The grid floor was made up of $.65-\mathrm{cm}$-diam stainless steel rods spaced $1.9 \mathrm{~cm}$ apart. A small hole just off-center in the front wall allowed the insertion of a drinking tube, with the orifice located approximately $5 \mathrm{~cm}$ into the chamber and $5 \mathrm{~cm}$ above the grid floor. A drinkometer circuit was formed between the water in the drinking tube and the grid floor. A $10-\mathrm{cm}$ loudspeaker for delivery of a tone $(15 \mathrm{sec}, 1,200 \mathrm{~Hz}, 90 \mathrm{~dB}$, from a Heathkit tone generator) was positioned at floor level behind the rear wall of each chamber. Both chambers were individually enclosed in a lighted, fan-ventilated, sound-attenuated box. Scrambled footshock, delivered through the grid floors of the chambers, was $1.6 \mathrm{~mA}, 3 \mathrm{sec}, 60 \mathrm{~Hz}$, and $330 \mathrm{~V} \mathrm{rms}$.

Surgery and Histology. The animals were anesthetized with pentobarbital $(50 \mathrm{mg} / \mathrm{kg})$ and implanted, unilaterally, with electrodes aimed at either the MRF or the DH, following System B of Pellegrino and Cushman (1967). The implantation coordinates for the MRF were $4 \mathrm{~mm}$ posterior from bregma, $2 \mathrm{~mm}$ lateral from midline, and $7.25 \mathrm{~mm}$ below the surface of the cortex. For the $\mathrm{DH}$, the coordinates were $3 \mathrm{~mm}$ posterior from bregma, $2.25 \mathrm{~mm}$ lateral from midline, and $3.50 \mathrm{~mm}$ below the surface of the cortex. 
Bipolar electrodes, obtained from Plastic Products, were constructed of insulated $.25-\mathrm{mm}$ stainless steel wire. The cutting of the electrode to proper length provided an uninsulated tip. Upon completion of the behavioral portion of the experiment, the animals were killed with pentobarbital overdose and perfused with saline and $11 \%$ Formalin solution, and electrode placements were confirmed with the method of Thomson, Beeman, Conger, Reeder, Steinhilb, Wittman, and DeVietti (1978).

Procedure. Following surgery, the animals were housed individually with food and water ad lib and were allowed a 7-10-day recovery period. They were then handled for approximately $5 \mathrm{~min}$. On each of the next 3 successive days, they were placed individually into one of the two chambers for a 10-min session. Some of the animals in each of the implanted groups were given two presentations of the 15-sec tone on each day (beginning at Minutes 2 and 7 of the session) for a total of six presentations. The remaining animals were simply placed in the chambers for 10 min each day and were not presented the tone. Following the third session, the water was removed from the home cages and the animals were allowed to drink 110 licks from a drinking tube present in the chambers the next day. Following this drink training session, water was returned to the home cages. On Day 5 , the animals were fearconditioned by initiating the 15 -sec tone 2 min into the session and presenting the footshock during the last $3 \mathrm{sec}$ of the tone. The animals were removed from the chambers $33 \mathrm{sec}$ after the offset of the tone and footshock and were returned to the home cage, from which the water was again removed.

The animals were tested, 24-h thirsty, the following day. Testing was started by gently touching the animal's nose to the drinking tube and releasing it near the rear of the chamber. After the animal had completed 100 licks on the drinking tube, the tone was automatically presented and remained on until the animal completed an additional 10 licks on the drinking tube. The time (in seconds) for the animal to complete 10 licks in the presence of the tone was automatically recorded and served as data for analysis. Animals not drinking within $600 \mathrm{sec}$ after the tone onset were removed from the apparatus and assigned a score of 600 .

Following the collection of data from these animals, we included an additional group of 13 animals implanted in the DH in a subsequent replication. These animals were treated identically to those described above, with the single exception that a total of 12 preexposure trials were administered, 4/day, in the three preexposure sessions. These trials were initiated at Minutes 2, 4, 6, and 7 of the 10-min session.

\section{Results and Discussion}

Figure 1 shows the locations of the electrode tips found to be located in the MRF and DH. Inspection of this figure reveals a fairly even distribution of electrode placements in the various subgroups formed on the basis of the number of preexposure trials administered. The histology revealed that one animal in each of the four groups formed by the factorial combination of implanted site and zero or six preexposure trials had incorrect electrode placements, whereas all DH animals given 12 preexposure trials were implanted correctly.

The latencies to drink in the presence of the test tone of animals with electrode placements confirmed to be in the MRF or DH were transformed to logarithms and are presented in Figure 2. A $2 \times 2$ unweighted means analysis of variance was performed on the data obtained from the original groups (i.e., the MRF and DH animals given zero or six preexposure trials). Both the main effects of implanted site and preexposure trials were significant sources of

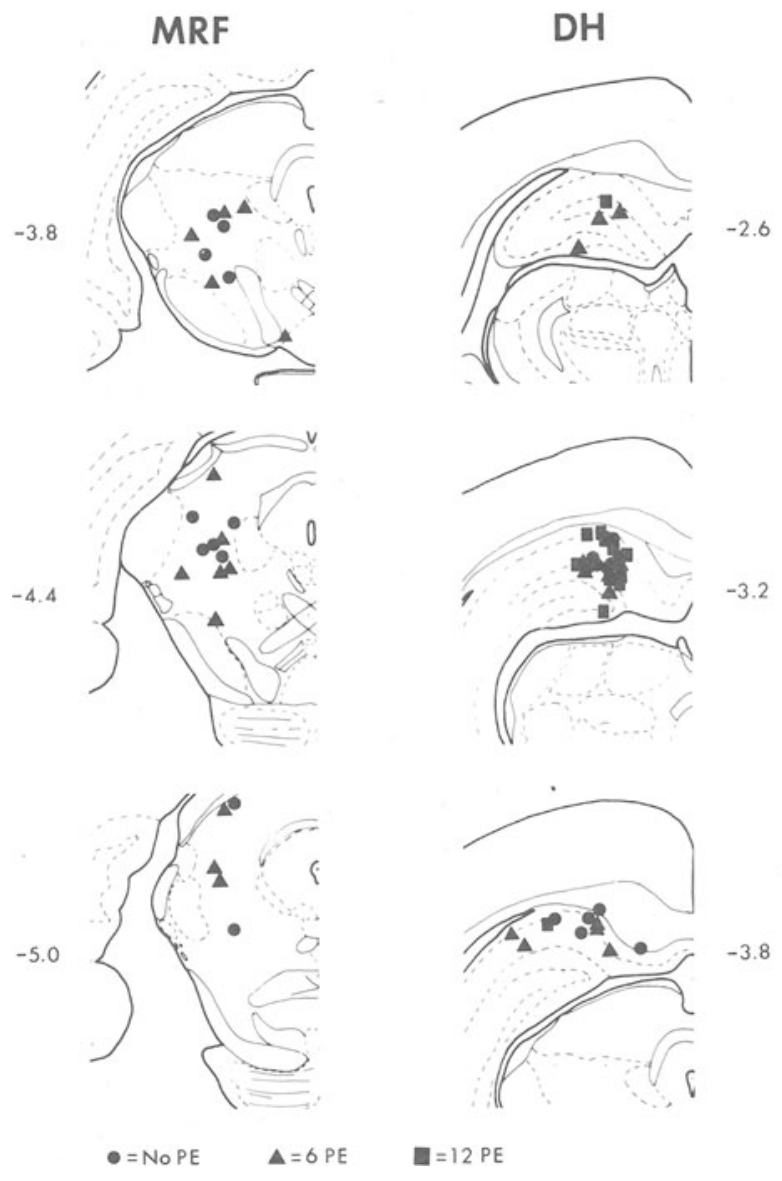

Figure 1. Electrode placements in MRF and DH animals. The distance (in $\mathrm{mm}$ ) posterior to bregma is shown along the side of the figure. Sections taken from Pellegrino and Cushman (1967).

variance $[\mathrm{Fs}(1,45)=9.69$ and 7.85 , respectively; ps $<.01$ ]. The interaction between these two variables was also statistically reliable $[F(1,45)=6.99$, $p<.02]$. The analysis of the simple main effects of this interaction confirmed the impression gained in viewing Figure 2 that latent inhibition was obtained in MRF animals, since those given six preexposure trials had shorter drink latencies relative to those not given preexposure $(p<.01)$. However, the comparison between the zero and six preexposure groups implanted in the DH failed to achieve statistical significance $(p>.05)$. Comparisons between MRF and DH groups revealed that, in groups given six preexposure trials, MRF animals had shorter drink latencies $(p<.01)$, but no difference was obtained between the groups given no preexposure $(p>.05)$. Latent inhibition was demonstrated in the DH animals given 12 preexposure trials, since their drink latencies were reliably shorter than those of the DH group given no preexposure $[\mathrm{t}(22)=2.21, \mathrm{p}<.05]$.

These findings replicate previous reports from our laboratory (DeVietti et al., 1980; DeVietti, Wittman, Emmerson, \& Thatcher, 1981) that six preexposure 


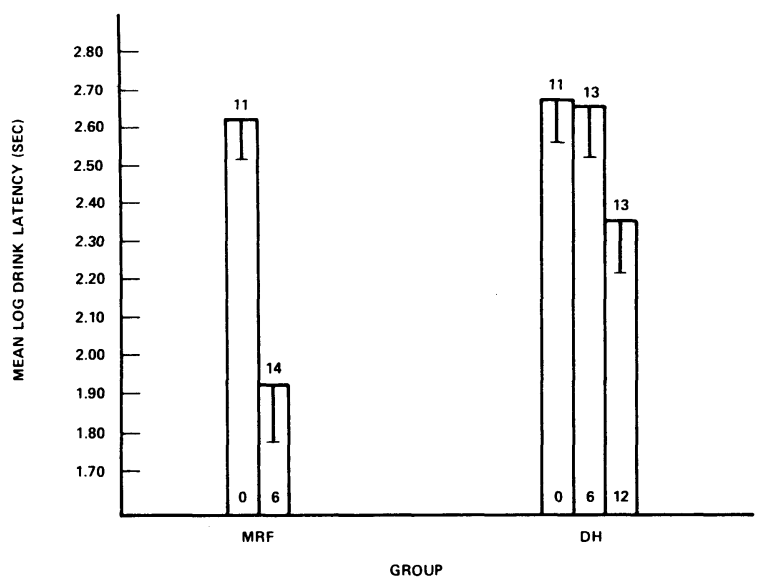

Figure 2. Mean log drink latencies in the presence of the tone in MRF and DH animals as a function of the number of preexposure trials. The number of animals in each group is shown above each mean, and the vertical line extending downward from each mean is the standard error.

trials result in latent inhibition in rats unilaterally implanted in the MRF. However, animals implanted in the DH failed to show latent inhibition when given six preexposure trials, although the effect was obtained in DH animals when the number of preexposure trials was increased to 12 . Thus, with six preexposure trials, it appears that the mere presence of an electrode in the DH is sufficient to disrupt latent inhibition. This result is consistent with, and extends the results of, previous reports demonstrating a disruption of latent inhibition with extensive, bilateral removal of the DH (Ackill et al., 1969; Solomon \& Moore, 1975).

\section{EXPERIMENT 2}

One interpretation of the finding that electrode implantation in the DH disrupts latent inhibition when 6 , but not 12 , preexposure trials are administered is that the DH is crucial to the demonstration of latent inhibition when few preexposure trials are administered. Another possibility is that the electrode located in the DH may have resulted in seizure activity that modified the impact of the preexposure trials. Unfortunately, the fact that latent inhibition was disrupted by 6 , but not 12 , preexposure trials appears to fit equally well with either explanation, as it is well known that increasing the number of preexposure trials increases latent inhibition (Lantz, 1973; Lubow, 1973). Also, increasing the number of preexposure trials to 12 would increase the probability of the animal's experiencing a greater number of preexposure trials, assuming that the DH animals were not in constant seizure.

We were not aware of any abnormal behavior in the DH animals during the course of the experiment that might have signaled the presence of seizure activity, for example, "wet-dog shakes" (Bloom, Segal,
Ling, \& Guillemin, 1976) or "catatonic states" (Andy \& Akert, 1955; DeVietti, 1964; MacLean, 1954). However, we thought it necessary to determine whether the presence of an electrode tip in the dorsal hippocampus, under our conditions, resulted in seizure activity present during the preexposure trials.

\section{Method}

Animals and Apparatus. The animals were 12 rats of the same stock and sex as described previously and were approximately 110 days of age. These animals had previously been fear-conditioned to the tone in a separate experiment and were unimplanted prior to their use in the present experiment. A Gilson polygraph was used to record EEG activity on a strip chart. Following initial recording during preexposure tones, a Pulsar 6 bp stimulator was used to induce seizure activity. The stimulation parameters were a 2-sec train of $330-\mathrm{Hz} 1-\mathrm{msec}$ biphasic rectangular pulses with a $1-\mathrm{msec}$ interpulse interval. The intensity ranged from 10 to $100 \mu \mathrm{A}$.

Surgery and Histology. The animals were implanted with electrodes aimed at the DH with the same coordinates used in Experiment 1 , and a baby clothes snap (Prims No. 0) was placed in one ear to serve as a reference electrode. At the conclusion of the experiment, the animals were perfused and electrode placement was confirmed, as described in Experiment 1.

Procedure. Following a 7-10-day recovery period after surgery, continuous EEG recordings were taken between the bipolar electrode and the reference electrode for $10 \mathrm{~min}$ on each of 3 successive days. The 15-sec tone was presented twice, beginning at Minutes 2 and 7 of each daily session. The following day, seizure thresholds were established by stimulation through the bipolar electrode, starting at $10 \mu \mathrm{A}$ and increasing in $10-\mu \mathrm{A}$ increments up to a maximum of $100 \mu \mathrm{A}$. At least $3 \mathrm{~min}$ elapsed between successive stimulations.

\section{Results and Discussion}

The histology revealed that the electrode tips were located in the DH of each animal and that the distribution was similar to that of the DH animals reported in Experiment 1.

Visual inspection of the EEG recordings failed to reveal any evidence of seizure activity in any animal at any time during the three preexposure sessions.

Seizure activity was induced by electrical stimulation of the $\mathrm{DH}$ in 8 of the 12 animals, with the intensity of stimulation necessary to induce seizure ranging between 30 and $100 \mu \mathrm{A}$. Four animals failed to show any seizure activity up to the maximum $100 \mu \mathrm{A}$ employed, although, at this intensity, there were obvious behavioral indications that the animals were receiving stimulation (e.g., forced movement). Figure 3 shows

A

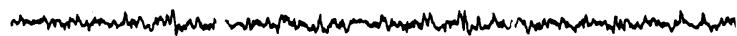

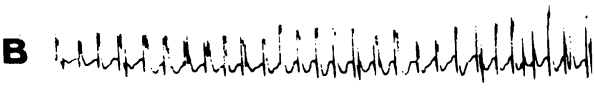
$\prod_{1 \mathrm{Sec} .} 0.5 \mathrm{mv}$

Figure 3. EEG activity in one animal recorded during the presentation of the tone (A) and immediately following stimulation of the DH (B). 
the EEG of a typical animal during a preexposure session. Also shown in this figure is seizure activity in this animal induced by $100 \mu \mathrm{A}$ of stimulation.

This experiment demonstrates that, under our conditions, the placement of an electrode in the $\mathrm{DH}$ is unlikely to result in seizure activity, present during the presentation of preexposure trials, that might explain the results of Experiment 1.

\section{GENERAL DISCUSSION}

Experiment 1 showed that the presence of an electrode in the DH disrupted latent inhibition when 6 preexposure trials were given, whereas, with 12 preexposure trials, latent inhibition was obtained. The results of Experiment 2 suggested that this effect was not likely due to seizure activity resulting from electrode implantation into the $\mathrm{DH}$. We suggest that these data favor the view that the integrity of the DH is essential to the development of latent inhibition when a small number of preexposure trials are given.

The mere presence of an electrode in the $\mathrm{DH}$ would seem to be a far more subtle manipulation than the extensive bilateral destruction of $\mathrm{DH}$ tissue used to disrupt latent inhibition in other studies (Ackil et al., 1969; Solomon \& Moore, 1975). However, both of these latter investigations used considerably more preexposure trials -30 and 450 , respectively-than did the present investigation. This, coupled with the fact that we were able to demonstrate a disruption of latent inhibition with 12 preexposure trials, may indicate a direct relationship between the number of preexposure trials given and the extent of $\mathrm{DH}$ damage necessary to block latent inhibition.

The goal of physiological psychology is to relate brain and behavior. Significant advances toward this goal are apparent from even a casual review of the literature. Regarding the hippocampus and restricting the approach to the implantation of electrodes or lesion techniques, evidence is accumulating that specific regions of this structure are involved in different behaviors. For example, Zornetzer, Boast, and Hamrick (1974) reported that long-term retention of passive avoidance was disrupted in mice bilaterally implanted in the dentate gyrus but that this effect was not obtained if the electrodes terminated in other regions of the hippocampus. However, it may be that this finding was related more to vascular damage resulting from the electrode implants than to the specific regions of the hippocampus in which the electrodes terminated (Boast, Reid, Johnson, \& Zornetzer, 1976). On the other hand, a large number of investigations have shown that specific lesions in hippocampal tissue differentially modify a wide variety of behaviors (Holdstock, 1972; Jarrard, 1976, 1978; Jarrard \& Becker, 1977; Kimura, 1958; Myhrer, 1975; Nadel, 1968). It is somewhat puzzling, therefore, that our work on the disruption of latent inhibition with the mere presence of an electrode in the $\mathrm{DH}$ has led us to conclude, so far, that there is no relationship between the region of the DH that the electrode tip is located and the disruption of latent inhibition. Considering the histology reported in Experiment 1, as well as extensive unpublished data from our laboratory, we have sampled many regions of the $\mathrm{DH}$ (e.g., cell fields CA1 and CA3, alveus, and the dentate and hippocampal gyri). Placements in all these regions have disrupted latent inhibition. Among other possibilities, this may mean that modification of any portion of the $\mathrm{DH}$ is sufficient to disrupt latent inhibition or, contrary to this equipotentiality view, that damage to more dorsal regions (e.g., alveus) is the critical factor. Obviously, our data do not permit a differentiation between these two possibilities.

Equally puzzling is the fact that MRF animals show latent inhibition when given six preexposure trials. With our surgical procedure, in order for the electrode tip to be placed in the MRF it must pass through the DH. Thus, MRF animals have the barrel of the electrode present in the DH, and, depending on the anterior-posterior coordinates, many MRF animals experienced greater DH interaction with the electrode than did many of the DH animals. Yet, MRF animals showed latent inhibition with six preexposure trials, whereas DH animals did not.

Since unimplanted animals show latent inhibition with six preexposure trials in our procedure (DeVietti et al., 1980), explanation of this paradox by assuming that damage to more ventral regions of the brain offsets the disruption of latent inhibition by DH damage seems unlikely. We thought perhaps the answer might lie in the fact that the surface of the electrode remaining in the DH in MRF animals was insulated, whereas electrode tips in the DH animals were uninsulated. We have tested this idea by implanting animals in the DH with either the electrode tips insulated or uninsulated and by giving six preexposure trials, as described in Experiment 1. In extensive parametric replications of this basic experiment, some of which gave encouraging results, we have, overall, been unable to demonstrate a difference in performance between these two groups. For the present, the safest statement would seem to be that the presence of an electrode tip, insulated or uninsulated, in the $\mathrm{DH}$ is sufficient to disrupt latent inhibition in animals given six preexposure trials. Thus, the paradox in the performance of MRF and DH animals remains.

\section{REFERENCE NOTE}

1. Salafia, R. W. Personal communication, November 1978.

\section{REFERENCES}

Ackil, J. E., Mellgren, R. L., Halgren, C., \& Frommer, G. P. Effects of CS preexposures on avoidance learning in rats with 
hippocampal lesions. Journal of Comparative and Physiological Psychology, 1969, 69, 739-747.

ANDY, O. J., \& AKERT, K. Seizure patterns induced by electrical stimulation of hippocampal formation in the cat. Journal of Neuropathology and Experimental Neurology, 1955, 14, 198-213.

Bloom, F., Segal, D., Ling, N., \& Guillemin, R. Endorphins: Profound behavioral effects in rats suggest new etiological factors in mental illness. Science, 1976, 194, 630-632.

Boast, C. A., Reid, S. A., Johnson, P., \& Zornetzer, S. F. A caution to brain scientists: Unsuspected hemorrhagic vascular damage resulting from mere electrode implantation. Brain $R e$ search, 1976, 103, 527-534.

DeVIetTi, T. L. Modification of hippocampal EEG with anticonvulsants. Unpublished master's thesis, University of Utah, 1964.

DeVietti, T. L., \& Larson, R. C. ECS effects: Evidence supporting state dependent learning in rats. Journal of Comparative and Physiological Psychology, 1971, 74, 407-415.

DeVietti, T. L., Wittman, T. K., \& Comfort, M. K. Rapid development of latent inhibition: No effect of implanted site. Psychological Reports, 1980, 47, 473-474.

DeVietti, T. L., Wittman, T. K., Emmerson, R. Y., \& Thatche R, D. O. Either stimulation of the mesencephalic reticular formation or a flashing light increases latent inhibition to a tone conditioned stimulus. Behavioral and Neural Biology, 1981, 32, 308-318.

Holdstock, T. L. Dissociation of function within the hippocampus. Physiology \& Behavior, 1972, 8, 659-667.

JARRARD, L. E. Anatomical and behavioral analysis of hippocampal cell fields in rats. Journal of Comparative and Physiological Psychology, 1976, 90, 1035-1050.

JARRARD, L. E. Selective hippocampal lesions: Differential effects on performance by rats of a spatial task with preoperative versus postoperative training. Journal of Comparative and Physiological Psychology, 1978, 92, 1119-1127.

JARrARD, L. E., \& Becker, J. T. The effect of selective hippocampal lesions on DRL behavior in rats. Behavioral Biology, 1977, 21, 393-404.
Kimura, D. Effects of selective hippocampal damage on avoidance behavior in the rat. Canadian Journal of Psychology, 1958, 12, 213-218.

LANTZ, A. E. Effect of number of trials, interstimulus interval, and dishabituation during CS habituation on subsequent conditioning in a CER paradigm. Animal Learning \& Behavior, 1973, 1, 273-277.

Lubow, R. E. Latent inhibition. Psychological Bulletin, 1973, 79, 398-407.

Lubow, R. E., \& Moore, A. U. Latent inhibition: The effect of non-reinforced preexposure to the conditioned stimulus. Journal of Comparative and Physiological Psychology, 1959, 52, 416-419.

MacLeAN, P. D. The limbic system and its hippocampal formation. Journal of Neurosurgery, 1954, 11, 29-44.

MYhre , T. Locomotor, avoidance, and maze behavior in rats with selective disruption of hippocampal output. Journal of Comparative and Physiological Psychology, 1975, 89, 759-777.

NADEL, L. Dorsal and ventral hippocampal lesions and behavior. Physiology \& Behavior, 1968, 3, 891-900.

Solomon, P. R., \& Moore, J. W. Latent inhibition and stimulus generalization of the classically conditioned nictitating membrane response in rabbits following dorsal hippocampal ablation. Journal of Comparative and Physiological Psychology, 1975, 89, 1192-1203.

Thomson, J. H., Beeman, F. J., Conger, G. L., Reeder, W. T., Steinhilb, K. R., Wittman, T. K., \& DeVietti, T. L. Videotape technology in the identification and storage of intracerebral electrode locations. Physiology \& Behavior, 1978, 20, 487-489.

Zornetzer, S. F., Boast, C., \& Hamrick, M. Neuroanatomic localization and memory processing in mice: The role of the dentate gyrus of the hippocampus. Physiology \& Behavior, $1974,13,569-575$.

(Manuscript received January 4, 1982; accepted for publication February 10, 1982.) 